\title{
Reflections on pseudo-scientific medicine
}

\section{Reflexões sobre medicina pseudo-científica}

Current image of physicians is confused with that of a scientist. Aiming at meeting this expectation, physicians in general avoid justifying their behaviors based on belief or faith, trying to bring logic arguments to their decisions. The problem is when we are faced with pseudo-scientific arguments favoring fanciful behaviors. I believe that the origin of this is the lack of understanding of the essence of the scientific thought.

Differently from what common sense believes, this essence is not in the presumption of understanding the universe, but rather in the humbleness of recognizing the uncertainty of our beliefs. This humbleness is presented on scientific minds as skepticism. The word skeptic comes from the Greek skepticos, which means "that who reflects, who questions, inspects". So a skeptic is not a boring person who does not believe in anything, but rather someone wishing to get close to a consistent truth.

Differently from skeptics, believers have already their truth and only try to find justifications to support it. Believers are presumptuous, dogmatic, while skeptics have the humbleness of recognizing their ignorance, which might be reduced in case there are evidences of the phenomenon. Based on skepticism, science has the role of building the puzzle of understanding the universe as from pieces which have the reliability of not concluding based on pseudo-evidences.

And what are pseudo-evidences? A common way of trying to convince someone is to present a mechanistic explanation. It is as if the explanation of the why could assure that something is true. However, the existence of a reason is not the proof of the crime. For example, some propose that vitamins have anti-cancer action because they have antioxidant effect. But it is not because oxidation predisposes to cancer that vitamins decrease the incidence of cancer. In fact, evidences with better methodological quality indicate the absence of this protective action ${ }^{1}$.

The second type of pseudo-science is to be fooled by real world illusions, caused by biases or by chance. One common type of bias is the confusion effect. It has been shown that European countries with large populations of storks have higher birth rates ${ }^{2}$. However, the connection storks-birth is measured by a third factor, the confusion factor. Countries with many storks have more rural cities where couples are more at ease to procreate. It was not the storks, but rather the types of cities.

To filter these illusions, the scientific method was created in early last century, which is a set of procedures to minimize illusions of universe observation in its natural state. The less the rigor of the scientific method, the more susceptible is the study to real world illusions. So, even the most fanciful behaviors have studies confirming them. What we need to do is to separate weed from wheat, choosing quality evidence to test our hypotheses.

I am not saying that it is wrong to adopt behaviors based on faith, because this is also part of life equation. Inadequate is to disguise such decisions as scientific. The mistake is to define some things as medicine.

Luis C. L. Correia

Escola Bahiana de Medicina e Saúde Pública

Hospital São Rafael

\section{REFERENCES}

1. Bjelakovic G, Nikolova D, Gluud C. ANtioxidant supplements to prevent mortality. JAMA. 2013;310(11):1178-9.

2. Matthews R. Storks deliver babies ( $\mathrm{p}=0.008$ ). Teaching Statistics. 2000;22(2):36-8. 